\title{
Rol de la resección paliativa en cáncer colorrectal estadio IV: Análisis de 177 pacientes*
} \author{
JAIME CONTRERAS P. ${ }^{1}$, CARLOS MELO L. ${ }^{1}$, DANIEL SOTO C. ${ }^{1}$ \\ Servicio y Departamento de Cirugía Hospital Clínico San Borja Arriarán. \\ 2 Escuela de Salud Pública. \\ Facultad de Medicina Universidad de Chile, Santiago, Chile.
}

Drs. GUILLERMO BANNURA C. ${ }^{1}$, ALEJANDRO BARRERA E. ${ }^{1}$, MiGUEL A. CUMSILlE G. ${ }^{2}$,

\begin{abstract}
Role of palliative excision of stage IV colon and rectal cancer. Analysis of 177 patients
\end{abstract}

Background: There is debate about the value of palliative excision of the primary tumor among patients with metastatic colorectal carcinoma. Aim: To assess the benefits of palliative excision, analyzing colon and rectal carcinomas separately. Material and Methods: Analysis of 86 patients with colon cancer aged $66 \pm 14$ years and 81 patients with rectal cancer aged $60 \pm 16$ years, in stage IV. Demographic and pathological variables were studied along with length of hospitalization, incidence of complications and actuarial survival. Results: No differences were observed in American Society of Anesthesiologists (ASA) classification, tumor size, proportion of undifferentiated or T3/T4 tumors and preoperative carcinoembrionic antigen. There was a predominance of locally advanced rectal tumors. In $44 \%$ of patients, a palliative excision was performed. No differences in rate of complications or operative mortality were observed between patients with colon or rectal tumors. Mean survival among patients with colon cancer subjected to palliative procedures was $15.2 \pm 13.2$ months compared with a survival of $4.4 \pm 3.7$ months of non operated patients $(\mathrm{p}<0.01)$. The figures for patients with rectal cancer were $20.5 \pm 19.6$ and $5.1 \pm 3.5$ months, respectively $(\mathrm{p}<0.01)$. Multivariate analysis showed that palliative excision was a significant predictor of survival. Conclusions: In this series of patients, palliative excision of colon and rectal tumors was associated with a significantly better survival and with acceptable rates of complications.

Key words: Colon cancer, rectal cancer, palliative excision.

\section{Resumen}

Antecedentes: Se ha debatido si la resección paliativa del tumor primario afecta la sobrevida en pacientes con un cáncer colorrectal metastásico. El objetivo de este estudio es evaluar esta hipótesis analizando por separado los tumores de colon y de recto. Pacientes y Método: Se estudia los pacientes en Estadio IV según el TNM 2002. Se analiza una serie de variables demográficas e histopatológicas por separado, así como la morbilidad operatoria y el tiempo de hospitalización. Se distingue tres tipos de patrón

*Recibido el 2 de Diciembre de 2008 y aceptado para publicación el 13 de Enero de 2009.

Correspondencia: Dr. Guillermo Bannura C.

Las Limas 1622, Santiago, Chile

E-Mail: gbannura@vtr.net 
metastásico dentro del estadio IV. El análisis de la sobrevida se realizó mediante el método de KaplanMeier y para establecer diferencias entre las curvas se utilizó el test log-rank. Resultados: Se trata de 96 pacientes con cáncer de colon y 81 con cáncer de recto en estadio IV, sin diferencias significativas en cuanto a ASA, tamaño tumoral, porcentaje de tumores indiferenciados, relación entre tumores T3/T4 y CEA preoperatorio, con predominio del patrón localmente avanzado en los tumores de recto $(p=0,04)$. Hubo un $44 \%$ de resecciones paliativas en colon y en recto, sin diferencias en la morbilidad (18\%) ni la mortalidad operatoria (1\%). El promedio de sobrevida en los pacientes de colon sometidos a una resección paliativa fue de 15,2 meses (DE: 13,18), y de 4,4 meses (DE: 3,66) en los no resecados (p < 0,0001). El promedio de sobrevida en los pacientes del recto resecados fue 20,5 meses (DE: 19,6), y de 5,06 meses (DE: $3,51)$ en el grupo no resecado $(\mathrm{p}<0,0001)$. En el análisis multivariado la resección paliativa y, en menor medida, la albúmina preoperatoria son los factores pronósticos más relevantes en colon y en recto, con una razón de disparidad de 4 y 5 veces, respectivamente $(\mathrm{p}<0,0001)$. Conclusión: La cirugía paliativa puede realizarse con bajos índices de morbimortalidad en pacientes aptos para soportar un procedimiento quirúrgico mayor. En los tumores de recto la resección paliativa se asocia con mayor morbilidad y mayor tiempo de hospitalización. La resección del tumor primario en el contexto de una enfermedad metastásica incurable prolonga en forma significativa la sobrevida de estos pacientes y debe intentarse en los pacientes con un riesgo razonable, exceptuando los casos con carcinomatosis peritoneal masiva y ascitis.

Palabras clave: Resección paliativa, cáncer de colon y recto, tumor primario, estadio IV.

\section{Introducción}

Aproximadamente el $20 \%$ de los pacientes portadores de un cáncer colorrectal se presentan al momento del diagnóstico en estadio IV, menos del $20 \%$ de ellos son candidatos a una resección quirúrgica con intención curativa y la expectativa de sobrevida global a 5 años no supera el $5 \%{ }^{1-3}$. La resección paliativa del tumor primario se considera habitualmente necesaria para controlar los síntomas de obstrucción, perforación y sangrado en pacientes aptos para soportar una cirugía mayor ${ }^{4}$. Se ha intentado identificar variables pronósticas que permitan predecir qué pacientes se podrían beneficiar de una intervención quirúrgica electiva. Para algunos autores la carga tumoral metastásica (medida con criterios no homogéneos), el grado de anemia, el CEA y la albúmina preoperatorios han demostrado cierta relevancia pronóstica, lo que no ha sido corroborado por otros ${ }^{4-6}$.

La mayoría de los trabajos analizan la seguridad del tratamiento quirúrgico y la sobrevida de los pacientes con enfermedad metastásica secundaria a un cáncer colorrectal considerados en forma global, es decir, como una entidad única. Sin embargo, la evidencia creciente demuestra que estos tumores tienen diferencias substanciales en cuanto al comportamiento biológico y la evolución clínica, lo que ha modificado la estrategia terapéutica, que es actualmente, radicalmente diferente en ambos tumores $^{7}$. En los últimos años la conducta agresiva de resección paliativa de los tumores de colon y de recto con enfermedad metastásica incurable ha sido discutida por la aparición de procedimientos o terapias alternativas menos invasivos ${ }^{8}$. ¿Se justifica la resección del tumor primario en un paciente con enfermedad diseminada? ¿Son comparables los resultados del tratamiento quirúrgico paliativo en los tumores de colon y de recto? Los beneficios potenciales de la resección paliativa -control de los síntomas, prevención de complicaciones y eventual mayor sobrevida- tiene como contrapeso la morbilidad y mortalidad asociada a una cirugía mayor. Este estudio es un análisis descriptivo de los resultados quirúrgicos de la resección paliativa del tumor primario en los pacientes en estadio IV analizando los tumores de colon y recto por separado.

\section{Material y Método}

Del registro prospectivo de pacientes intervenidos por un adenocarcinoma de colon y de recto desde enero de 1991 hasta diciembre de 2006 se estudia el grupo de pacientes en Estadio IV según la clasificación de la UICC conocida como TNM $2002\left(6^{\text {th }} \text { edición }\right)^{9}$. La etapificación se realizó mediante los antecedentes clínicos, exámenes por imágenes, hallazgos intraoperatorios y el estudio de la pieza operatoria o biopsia, según corresponda. Se incluye todos los pacientes estadio IV sintomáticos intervenidos en forma electiva ASA I a III aptos para soportar una laparotomía exploradora. Se excluye los resecados con intención curativa y los pacientes con un tumor de recto bajo sometidos a una resección local paliativa. Se analiza una serie de variables demográficas e histopatológicas por separado en los pacientes con tumores de colon (grupo 1) y de recto (grupo 2). La morbilidad operatoria y el tiempo de hospitalización según el 
tipo de intervención se utilizaron como una variable indirecta del impacto de la cirugía en la evolución de los pacientes. La cirugía no curativa se define por la irresecabilidad del tumor primario, la presencia de enfermedad residual local no resecable (R2), la carcinomatosis peritoneal y/o compromiso de los ganglios lumboaórticos y/o metástasis a distancia irresecables. Se define como resección paliativa (RP) la extirpación radical del tumor primario dejando enfermedad metastásica residual a distancia. El resto de las intervenciones se define como no resectivas (NR), incluyendo las derivaciones o by pass entre intestino delgado y colon o entre dos segmentos de colon, las ostomías y las laparotomías exploradoras. El antígeno carcinoembrionario (CEA) se consideró como variable continua y como variable categórica, considerando $5 \mathrm{ng} / \mathrm{ml}$ como límite del valor normal. El perfomance status del paciente se evaluó de acuerdo a la edad, el ASA y las enfermedades asociadas. La albúmina preoperatoria se trató como variable continua y categórica, considerando $3,8 \mathrm{gr} / \mathrm{dl}$ como "cut off". Un tumor ubicado entre el ciego y el ángulo esplénico se consideró como cáncer de colon derecho y desde allí hasta el promontorio como cáncer de colon izquierdo. Las lesiones ubicadas por debajo de los $15 \mathrm{~cm}$ del margen anal (promontorio) se consideran como cáncer del recto. En cuanto al patrón metastásico del estadio IV se distinguió entre 1) Metástasis a distancia (hígado, pulmón); 2) Carcinomatosis peritoneal y/o adenopatías lumboaórticas metastásicas y 3) Compromiso local avanzado irresecable o resecado en forma incompleta (R2), registrando sólo una manifestación de la enfermedad por paciente. La supervivencia se define en meses como sobrevida cruda, comparando los pacientes sometidos a una RP, o a una NR en ambos grupos.

Para el análisis descriptivo y el análisis univariado de asociaciones se utilizó la prueba del chi cuadrado de Pearson, el test de Wilcoxon (Mann-Whitney) $y$ la prueba $t$ de Student, según corresponda. El análisis de la sobrevida se realizó mediante el método de Kaplan-Meier y para establecer diferencias entre las curvas se utilizó el test log-rank. El análisis multivariado se efectuó mediante el modelo de regresión logística de Cox. Se consideró estadísticamente significativo un $\mathrm{p}<0,05$.

\section{Resultados}

Se incluyen todos los pacientes sintomáticos operados en forma electiva por un cáncer de colon o de recto en el período señalado $(n=676)$. La operabilidad global de la serie es $97 \%$ y la reseca- bilidad global es 78\%. Hubo 385 pacientes intervenidos en forma consecutiva por un adenocarcinoma de colon, de los cuales $97(25,1 \%)$ tenía enfermedad metastásica incurable al momento de la laparotomía. Un paciente con un tumor localmente irresecable de colon derecho sometido a una ileotransversoanastomosis fallece a los 20 días por progresión de la enfermedad, sin complicación quirúrgica. De los 291 tumores de recto operados en dicho período, $82(28,1 \%)$ casos correspondían a un estadio IV, de los cuales un paciente fallece por una embolia pulmonar masiva luego de múltiples complicaciones quirúrgicas a los 28 días de una resección paliativa (mortalidad operatoria de la cirugía paliativa en cáncer de colon y recto fue $1 \%$ y $1,2 \%$, respectivamente). De este modo, 96 pacientes con un cáncer de colon y 81 con un cáncer de recto en estadio IV constituyen el universo de este estudio. La localización de las lesiones corresponde a 50 casos de tumores de colon derecho, 46 de colon izquierdo, 22 del tercio superior del recto, 30 del tercio medio y 29 del tercio inferior. Las características demográficas y el tipo de cirugía efectuada se aprecian en la Tabla 1, incluido el principal patrón metastásico por paciente. No hubo diferencias en cuanto al ASA, el tamaño tumoral medido en los pacientes resecados $(7,1 \mathrm{~cm}$ (DE: 2,67$)$ en colon, y 6,65 (DE: 2,73 ) en recto; $p=0,44$ ), el porcentaje de tumores indiferenciados $(20 \%$ en colon y $22 \%$ en recto; $\mathrm{p}=0,91)$ y la relación entre tumores T3/T4 $(61 / 35$ en colon, y 46/35 en recto; $p=0,36)$. El CEA como variable continua tuvo un promedio de 40,53 $\mathrm{ng} / \mathrm{ml}$ en 64 pacientes con un tumor de colon (DE: 95,61 ; extremos 1,1-382) y un promedio de 72,61 en 62 pacientes con un tumor de recto (DE: 34,61 ; extremos 0,1-3272), diferencia que no alcanza significación estadística $(\mathrm{p}=0,07)$. Aunque los grupos son comparables, los pacientes con un cáncer de recto son más jóvenes, tienen un hematocrito preoperatorio promedio superior y muestran un mayor grado de irresecabilidad del tumor primario por compromiso local avanzado que los tumores de colon.

En el grupo de los pacientes con un tumor de colon se efectuó 42 (44\%) RP (20 colectomías derechas, 20 resecciones izquierdas y 2 colectomías totales), con 3 resecciones sincrónicas de metástasis hepáticas. No hubo diferencias en la tasa de resecciones según la localización del tumor (40\% en colon derecho, $48 \%$ en colon izquierdo). El patrón metastásico en el grupo resecado fue metástasis a distancia en 22 casos (52\%), carcinomatosis focal y/o adenopatías lumboaórticas en $19(45 \%)$ y tumor localmente avanzado en 1 (2\%). Entre las intervenciones NR destacan 22 derivaciones, 17 ostomías (8 ileostomías) y 15 laparotomías explora- 


\begin{tabular}{|c|c|c|c|c|c|}
\hline Variable & & $\begin{array}{l}\text { olon } \\
=96\end{array}$ & & $\begin{array}{r}\text { ecto } \\
=81\end{array}$ & $\mathbf{p}$ \\
\hline Edad promedio (DE) & 66,3 & $(13,94)$ & 60,3 & $(15,68)$ & 0,007 \\
\hline Género (mujer/hombre) & $50 / 46$ & & $39 / 42$ & & 0,60 \\
\hline Enfermedades asociadas (\%) & 48,9 & & 40,7 & & 0,27 \\
\hline Hematocrito promedio $*(\mathrm{DE})$ & 32,58 & $(6,75)$ & 34,53 & $(6,30)$ & 0,05 \\
\hline Albúmina promedio * (DE) & 3,69 & $(0,52)$ & 3,73 & $(0,60)$ & 0,62 \\
\hline $\mathrm{CEA}>5 \mathrm{ng} / \mathrm{ml} \mathrm{n} *(\%)$ & $39 / 64$ & $(61)$ & $44 / 62$ & $(71)$ & 0,23 \\
\hline Resección paliativa n (\%) & 42 & $(44)$ & 36 & $(44)$ & 0,92 \\
\hline $\begin{array}{l}\text { Estadio IV }(\%) \\
\text { - Metástasis a distancia } \\
\text { - Carcinomatosis y/o LN Lu-Ao }{ }^{\&} \\
\text { - Localmente avanzado }\end{array}$ & $\begin{array}{l}29 \\
54 \\
13\end{array}$ & $\begin{array}{l}(30) \\
(56) \\
(14)\end{array}$ & $\begin{array}{l}19 \\
39 \\
23\end{array}$ & $\begin{array}{l}(23) \\
(48) \\
(28)\end{array}$ & 0,04 \\
\hline
\end{tabular}

DE: desviación estándar; *Valores preoperatorio; ${ }^{\&}$ Linfonodos lumboaórticos con biopsia + para adenocarcinoma.

doras. Hubo 18 (19\%) pacientes (6 RP y 12 NR) con una o más complicaciones, destacando la infección del sitio operatorio (ISO) en 7 casos; íleo prolongado en 5; neumopatía en 3; 1 paciente con infección del tracto urinario (ITU), una sepsis por catéter; una colección pélvica y una fístula intestinal, ambas de manejo médico y un paciente requiere una resección de intestino delgado por una trombosis mesentérica parcelar con buena evolución posterior.

En el grupo de pacientes con un tumor de recto se realizó $36(44,4 \%)$ RP (22 resecciones anteriores, 11 abdominoperineales, 2 Hartmann y 1 panproctocolectomía), con 2 resecciones sincrónicas de metástasis hepáticas. El patrón metastásico en el grupo resecado fue metástasis a distancia en 17 (47\%), carcinomatosis peritoneal focal y/o adenopatías lumboaórticas en 17 (47\%) y tumor localmente avanzado en $2(5,5 \%)$. Entre las intervenciones NR hubo 42 ostomías (10 ileostomías) y 3 laparotomías exploradoras. Hubo 18 (22,2\%) pacientes (14 RP y 4 NR) con una o más complicaciones, destacando el íleo prolongado en 3 casos, ISO en 4, ITU en 4, una fístula rectovaginal, una fístula ascítica, una peritonitis de causa no precisada, una neumopatía, una necrosis de la colostomía y dos casos de fístula anastomótica. Hubo 4 reoperaciones correctoras con buena evolución posterior: aseo peritoneal (1), recolostomía (1), Hartmann (1) y colostomía (1), dos en el grupo RP y dos en el grupo NR.

Al comparar los pacientes según el tipo de cirugía efectuada, podemos apreciar que no existen diferencias en las características demográficas entre el grupo sometido a una resección radical paliativa y el grupo no resecado, tanto en colon como en recto, salvo un menor hematocrito preoperatorio promedio en los pacientes no resecados en los tumores de recto. La morbilidad global fue similar en los pacientes con tumores de colon (18/96) y de recto $(18 / 81)(p=0,57)$. El tiempo de hospitalización global fue 10,30 días (DE: 5,3; extremos 4-28) en los tumores de colon, y 12,83 (DE: 12,5; extremos 1-64) en los tumores de recto $(p=0,035)$. Esta diferencia se debe a la mayor morbilidad y mayor tiempo de hospitalización de las RP en los pacientes con tumores del recto comparado con las intervenciones NR, lo que es estadísticamente muy significativo (Tabla 2).

Doce pacientes en el grupo colon (11 resecados) y 8 en el grupo recto (5 resecados) fueron sometidos a quimioterapia adyuvante paliativa, mientras que 9 pacientes del grupo recto (todos resecados) recibieron radioquimioterapia postoperatoria. La sobrevida promedio global de la serie fue 10,43 meses (DE: 13,06; extremos 1-90), cifra que es similar en ambas localizaciones: 9,17 meses (DE: 10,55) en colon, y 11,92 (DE: 15,45) en recto $(p=0,93)$. La sobrevida promedio de los pacientes resecados fue 17,7 meses para la serie global, cifra que alcanza a 15,75 meses (DE: 11,25) en los pacientes resecados con patrón de metástasis a distancia, 18,41 meses (DE: 15,95) en los casos con carcinomatosis focal y 19,16 meses (DE: 20,73) en los tumores localmente avanzados $(\mathrm{p}=0,004)$.

El promedio de sobrevida en los pacientes del grupo 1 (colon) sometidos a una resección paliativa fue de 15,2 meses (DE: 13,18), y 4,4 meses (DE: $3,66)$ en los no resecados $(p<0,0001)$. El promedio de sobrevida en los pacientes del grupo 2 (recto) 
Tabla 2. Características de los pacientes con cáncer de colon y recto estadio IV según tipo de cirugía. Morbilidad y hospitalización

\begin{tabular}{|c|c|c|c|c|c|c|}
\hline \multirow[b]{2}{*}{ Variable } & \multicolumn{3}{|c|}{ Colon $n=96$} & \multicolumn{3}{|c|}{ Recto $n=81$} \\
\hline & $\mathbf{R P}$ & NR & $\mathbf{p}$ & $\mathbf{R P}$ & NR & $\mathbf{p}$ \\
\hline Edad promedio & 64,78 & 67,5 & 0,34 & 58,22 & 61,97 & 0,28 \\
\hline Género (mujer/hombre) & $22 / 20$ & $28 / 26$ & 0,96 & $20 / 16$ & $19 / 26$ & 0,23 \\
\hline Enfermedades asociadas \% & 50 & 48,1 & 0,85 & 38,9 & 42,2 & 0,76 \\
\hline Hematocrito promedio & 32,16 & 32,90 & 0,59 & 36,52 & 32,93 & 0,009 \\
\hline Albúmina promedio & 3,76 & 3,63 & 0,24 & 3,85 & 3,64 & 0,12 \\
\hline $\mathrm{CEA}>5 \mathrm{ng} / \mathrm{ml} /$ total & $20 / 30$ & $15 / 34$ & 0,37 & $24 / 32$ & $20 / 30$ & 0,47 \\
\hline Morbilidad & $6 / 42$ & $12 / 54$ & 0,32 & $14 / 36$ & $4 / 45$ & 0,001 \\
\hline Hospitalización prom (ds) & 10,86 & 9,87 & 0,36 & 17,58 & 9,04 & 0,001 \\
\hline
\end{tabular}

$\mathrm{RP}=$ resección paliativa. $\mathrm{NR}=$ técnicas no resectivas.

Tabla 3. Análisis multivariado (regresión logística de Cox) de la sobrevida global en pacientes con cáncer de colon y de recto estadio IV

\begin{tabular}{lcccccc}
\hline Variable* & \multicolumn{3}{c}{ Colon } & \multicolumn{2}{c}{ Recto } \\
$\mathbf{9 5 \%}$ IC & RD & $\mathbf{p}$ & RD & $\mathbf{9 5 \%}$ IC & $\mathbf{p}$ \\
\hline Edad (<65 años) & 1,007 & $0,99-1,02$ & 0,36 & 1,007 & $0,99-1,02$ & 0,38 \\
Género (mujer) & 0,69 & $0,45-1,07$ & 0,10 & 1,66 & $0,97-2,85$ & 0,06 \\
Albúmina (<3,8 gr/dl) & 0,60 & $0,38-0,93$ & 0,023 & 0,50 & $0,29-0,85$ & 0,011 \\
Localización (Colon der) (recto bajo) & 0,56 & $0,30-1,04$ & 0,06 & 1,08 & $0,57-2,05$ & 0,79 \\
Operación (no resección) & 4,06 & $2,20-7,50$ & $<0,0001$ & 5,14 & $2,66-9,94$ & $<0,0001$ \\
\hline
\end{tabular}

*Se señala la variable teórica de menor sobrevida.

resecados fue 20,5 meses (DE: 19,6), y 5,06 meses (DE: 3,51) en el grupo no resecado $(p<0,0001)$. $\mathrm{Al}$ introducir en el modelo de Cox el conjunto de variables posibles de impactar en la sobrevida global se aprecia que la resección paliativa y, en menor medida, la albúmina preoperatoria son los factores pronósticos más relevantes en ambas localizaciones (Tabla 3). Así, los pacientes resecados sobreviven 4 veces más en el grupo 1 y 5 veces más en el grupo 2 y los pacientes con una mejor albúmina preoperatoria prolongan la sobrevida 1,6 veces más en el grupo 1 y 2 veces más en el grupo 2 . Al excluir los pacientes sometidos a terapia adyuvante se mantienen las diferencias entre las RP y NR.

En el grupo de pacientes con un tumor de colon, el 20\% sobrevive más de 12 meses (16 RP y 3 NR), el 10\% sobrevive más de 24 meses (todos resecados) y el 5\% más de 36 meses (lo que representa el $10 \%$ de los pacientes resecados). Al corte del estudio hay 5 pacientes vivos a los $61,37,31,29$ y 18 meses de seguimiento, respectivamente, 4 con me- tástasis hepáticas múltiples y el restante (linfonodos positivos de la cadena lumboaórtica) completa 29 meses en control sin enfermedad aparente (Figura 1). En el grupo de pacientes con un tumor de recto, el $27 \%$ sobrevive más de 12 meses (sólo 1 paciente NR), el 11\% más de 24 meses y el 8,6\% más de 36 meses (todos RP, lo que representa el 19,4\% de los resecados). Al momento del corte hay 4 pacientes vivos a los 90, 81, 45 y 12 meses de seguimiento, respectivamente, 3 con metástasis a distancia y el restante (linfonodos intercavo-aórticos positivos) completa 81 meses de observación sin evidencia de enfermedad (Figura 2).

\section{Discusión}

Aunque en esta serie no hay grandes diferencias entre los pacientes portadores de un cáncer de colon metastásico comparado con los estadio IV de recto y la tasa de resecabilidad global es similar 


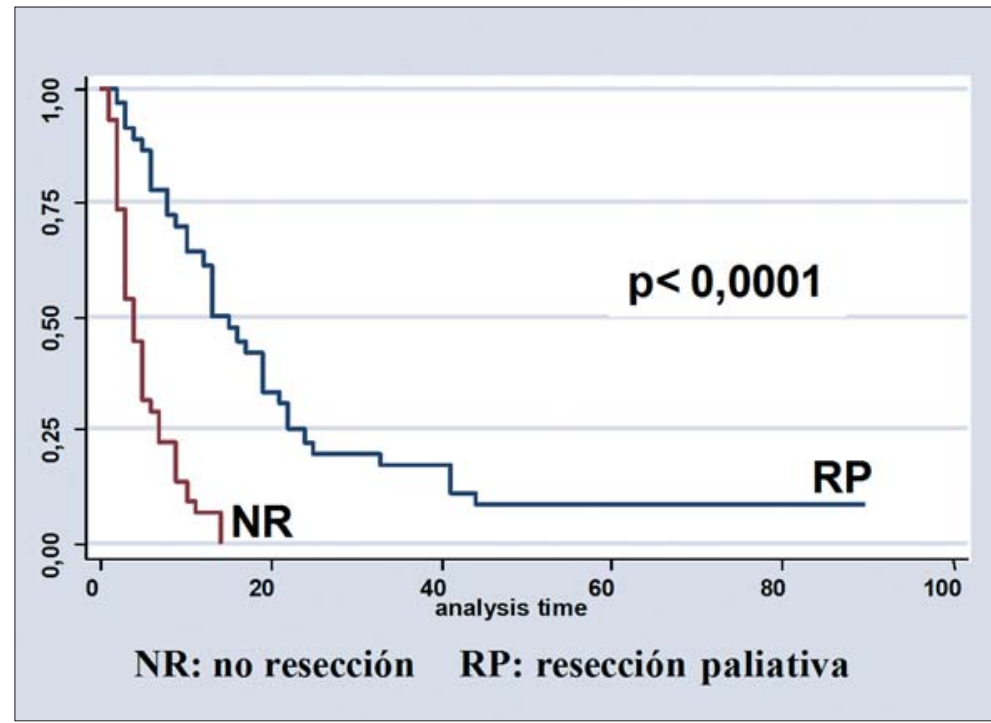

Figura 1. Kaplan-Meier. Sobrevida en cáncer de colon Estadio IV según tipo de cirugía.

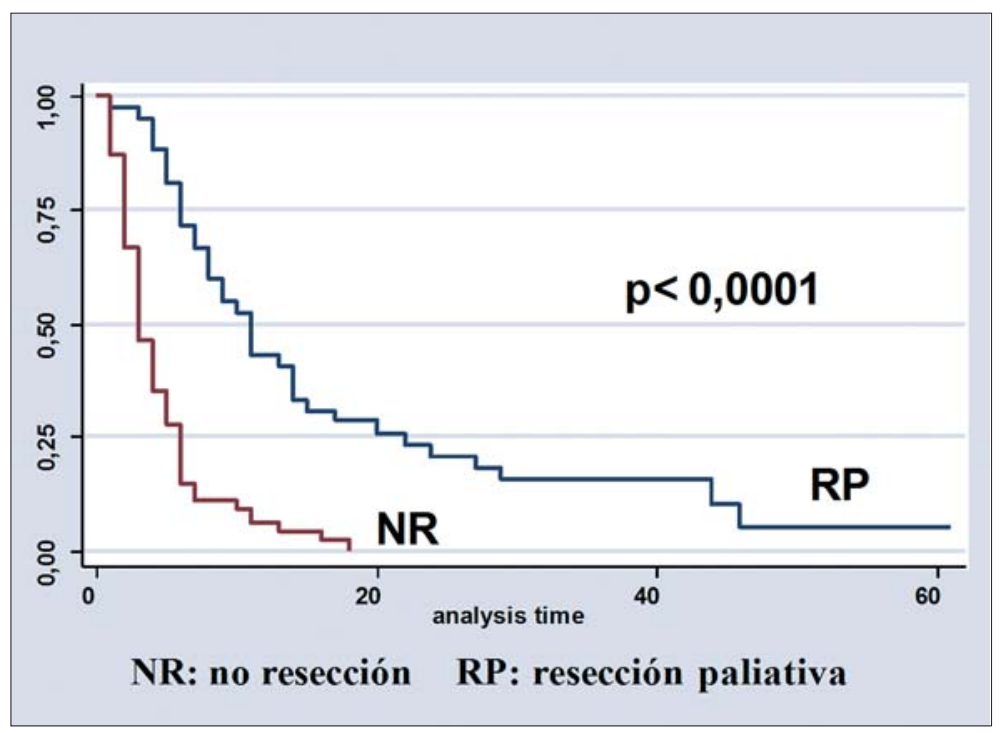

Figura 2. Kaplan-Meier. Sobrevida en cáncer de recto Estadio IV según tipo de cirugía. e independiente de la localización de la lesión, los pacientes con tumores de recto son más jóvenes, tienen un hematocrito promedio preoperatorio más elevado y el compromiso local avanzado es mayor que en los pacientes con tumores de colon. Esto no se explica por diferencias en la magnitud de la carga tumoral al momento de la cirugía, puesto que otros parámetros como la albúmina y el CEA, así como la distribución por género y las condiciones co-mórbidas, son muy similares entre ambos grupos. Desgraciadamente no es posible comparar estos resultados con los publicados, puesto que la mayoría de los autores analizan los pacientes en forma global bajo el rubro tumores colorrectales en estadio IV ${ }^{4-6,10-12}$. La tasa de tumores indiferenciados es el doble de la serie global de nuestra Institución $^{13}$.

El porcentaje de resecciones paliativas del tumor primario en esta serie (44\%) es algo inferior a otras publicaciones extranjeras que promedian el $66 \%{ }^{14}$, lo que pudiera explicarse en parte por diferencias en la oportunidad del diagnóstico $\mathrm{y}$, por tanto, de tratamiento, lo que se corrobora con la mayor frecuencia de estadios IV en nuestra serie ( 26\%). Por otro lado, la tasa de mortalidad del 1\% se compara favorablemente con la mayoría de las 
series publicadas, que fluctúan entre $2 \%$ y $15 \%$,6,1012,15,16, lo que refleja una adecuada selección de los pacientes que van a ser sometidos a una cirugía paliativa (Tabla 4). La morbilidad de esta serie no es diferente a la serie de pacientes intervenidos con intención curativa en nuestra institución y es similar a las series publicadas ${ }^{4,6,10-12,15,16}$. Al separar los tumores de colon y de recto podemos apreciar que en ambas localizaciones la resección del tumor primario es una decisión intraoperatoria determinada por la factibilidad de realizarla, puesto que no se aprecian diferencias demográficas significativas entre el grupo sometido a RP y los NR, similar a otros autores ${ }^{6,14,15}$. En otras series, que analizan globalmente los tumores colorrectales, existen diferencias significativas en cuanto al CEA preoperatorio, localización, carga tumoral, grado de diferenciación tumoral y compromiso linfonodal ${ }^{10-12}$, entre los pacientes sometidos a una RP respecto de los no resecados. En nuestra serie la decisión de efectuar una resección del tumor primario tiene un impacto clínico en términos de mayor morbilidad y mayor tiempo de hospitalización sólo en los tumores de recto, lo que no es menor en un paciente que ya tiene un sobrevida limitada. Anatómicamente, el recto no puede ser motivo de una derivación interna, por lo que la única solución no resectiva para esta localización es la ostomía. Similar a otros autores ${ }^{13-16}$, no existen diferencias en la morbilidad global entre las RP y la NR, lo que probablemente se explica porque la mayor envergadura de la RP se compensa con la mayor carga tumoral de los pacientes sometidos a una intervención NR.

En nuestra experiencia y de la mayoría de los autores ${ }^{4-6,10-12,14-16}$ la RP prolonga la sobrevida de los pacientes portadores de un tumor con enfermedad metastásica incurable (Tabla 4). El análisis multivariado confirma que la resección paliativa es el factor pronóstico más relevante en la sobrevida observada en estos pacientes, seguida de la albúmina preoperatoria. La albúmina se ha considerado como un indicador del impacto y la magnitud de la carga tumoral en el paciente portador de una neoplasia avanzada de colon o de recto y para algunos autores en combinación con el CEA preoperatorio podría servir para identificar los mejores candidatos a un $\mathrm{RP}^{5}$. El análisis por separado de los tumores de colon y de recto nos señala que este beneficio es significativo en ambos grupos y, aunque tiene un costo en cuanto a morbilidad en los tumores de recto, es más destacado aún que en los pacientes con un tumor de colon. Se desprende de este estudio que la resección paliativa tiene un lugar destacado en el manejo del paciente con un cáncer de colon o de recto con enfermedad avanzada incurable. Esta recomendación no puede ser taxativa dadas las limitaciones de un estudio retrospectivo y es posible que, a pesar de las variables estudiadas, los grupos no sean comparables. En efecto, es razonable suponer que exista un sesgo de selección en los pacientes sometidos a cirugía y, particularmente, la carga tumoral no sea similar en ambos grupos. Los criterios para medir la carga tumoral son muy variables en las diferentes series publicadas $\mathrm{y}$, hasta donde sabemos, ningún estudio ha evaluado estos factores en forma prospectiva y al azar para sacar una conclusión definitiva. Si bien el compromiso metastásico en el hígado puede ser evaluado en forma más o menos precisa por el estudio de imágenes, el estadio IV incluye pacientes muy heterogéneos en los cuales la medición es muy difícil. Es probable que en nuestra serie, por ejemplo, los pacientes con carcinomatosis focal que fueron resecados tuvieran una carga tumoral muy inferior a los NR con el mismo patrón metastásico y, hecho no considerado en esta revisión, el $20 \%$ de los pa-

Tabla 4. Morbimortalidad y sobrevida en pacientes operados por un cáncer colorrectal metastásico. Series publicadas 2003-2007

\begin{tabular}{llccccc}
\hline Autor & $\mathbf{A} \mathbf{n}^{\text {ref }}$ & $\mathbf{n}$ & $\begin{array}{c}\text { Morbilidad } \\
\mathbf{\%}\end{array}$ & $\begin{array}{c}\text { Mortalidad } \\
\mathbf{\%}\end{array}$ & $\begin{array}{c}\text { Sobrevida promedio (meses) } \\
\text { RP/NR* }\end{array}$ & $\begin{array}{c}\text { (m } \\
\mathbf{p}\end{array}$ \\
\hline Ruo & $03^{10 \&}$ & 127 & 20,5 & 1,6 & $16 / 9$ & $<0,001$ \\
Stelzner & $05^{6 \&}$ & 186 & $\mathrm{~ns}$ & 11 & $11,7 / 5,2$ & 0,0002 \\
Beham & $06^{19}$ & 67 & 4,5 & 2,8 & $18,1 / 7,8$ & $<0,001$ \\
Konyalian & $06^{14}$ & 109 & 14 & 6 & $12,5 / 4,6$ & $<0,001$ \\
Costi & $07^{11}$ & 130 & 32 & 10,7 & $9 / 3$ & $<0,001$ \\
Yun & $07^{12}$ & 376 & 3 & 2 & $18,5 / 5,3$ & $<0,001$ \\
Bannura & 08 & 177 & 18 & 1 & $17,7 / 4,6$ & $<0,0001$ \\
\hline
\end{tabular}

*RP $=$ resección paliativa; $\mathrm{NR}=$ no resección. ${ }^{\&}$ Serie de pacientes asintomáticos. 
cientes tienen más de una manifestación de la enfermedad diseminada. A pesar de las limitaciones señaladas, podemos concluir que la resección paliativa del tumor primario estaría justificada en pacientes con enfermedad incurable toda vez que sea factible en un paciente de riesgo quirúrgico normal si el equipo tratante muestra cifras de morbimortalidad equivalentes a la cirugía resectiva con intención curativa. La importancia de las terapias adyuvantes, que indudablemente pueden impactar en la sobrevida de estos pacientes, requiere de un estudio prospectivo multicéntrico y está fuera del alcance de este estudio.

El rol de la cirugía paliativa en los pacientes asintomáticos o con escasos síntomas, en los cuales la enfermedad metastásica es un hallazgo, es motivo de controversia ${ }^{10}$ y no se analiza en esta serie compuesta exclusivamente por pacientes sintomáticos. Varios estudios han propuesto un manejo no quirúrgico de estos pacientes con resultados muy variables ${ }^{8,12,17,18}$. Los métodos disponibles incluyen la quimioterapia, con o sin radioterapia, la decomprensión endoscópica con prótesis y la recanalización del tumor con láser, los que pueden combinarse de acuerdo con la localización del tumor.

En conclusión, la cirugía paliativa electiva tiene un rol destacado en el tratamiento quirúrgico de los pacientes con un cáncer de colon o de recto en estadio IV. Es capaz de controlar en forma satisfactoria los síntomas derivados de la obstrucción y de la hemorragia. La cirugía paliativa puede realizarse con bajos índices de morbimortalidad en pacientes aptos para soportar un procedimiento quirúrgico mayor. La resección del tumor primario en el contexto de una enfermedad metastásica incurable prolonga en forma significativa la sobrevida de estos pacientes y debe intentarse en los pacientes con un riesgo razonable, exceptuando los casos con una carcinomatosis peritoneal masiva y ascitis ${ }^{11,19} \mathrm{y}$, probablemente, los pacientes con extenso compromiso del hígado $(>75 \%)^{14}$. La RP en los tumores de recto tiene un impacto negativo en cuanto a la morbilidad y el tiempo de hospitalización, por lo que debe evaluarse por separado del cáncer de colon. Estudios prospectivos multicéntricos que incluyan encuestas de calidad de vida se requieren para validar estas conclusiones ${ }^{20}$. Una definición universal del patrón metastásico en pacientes en estadio IV es fundamental para comparar resultados.

\section{Referencias}

1. American Cancer Society. Cancer facts and Figures 2002. Available: http://www.cancer.org.docroot.

2. Mella J, Biffin A, Radcliffe AG, Stamatakis JD, Steele
RJ. Population based audit of colorectal cancer management in two UK health regions. Colorectal Cancer Working Group, Royal College of Surgeons of England Clinical Epidemiology and Audit Unit. Br J Surg 1997; 84: 1731-1736.

3. The Cancer Registry of Norway (2000). Cancer in Norway 2003. http://kreftreregister.no/forekomst-orgovelevelse-2000/cin2000.pdf

4. Makela J, Haukipuro K, Laitinen S, Kairaluoma MI. Palliative operations for colorectal cancer. Dis Colon Rectum 1990; 33: 846-850.

5. Dixon M, Haukoos JS, Udani SM, Naghi JJ, Arnell T, Kumar RR, et al. Carcinoembriogenic antigen and albumin predict survival in patients with advanced colon and rectal cancer. Arch Surg 2003; 138: 962-966.

6. Stelzner S, Hellmich G, Koch R, Ludwig K. Factors predicting survival in stage IV colorectal carcinoma patients after palliative treatment: a multivariate analysis.

7. Li M, Li JY, Zhao AL, Gu J. Colorectal cancer or colon and rectal cancer? Clinicopathological comparison between colonic and rectal carcinomas. Oncology 2007; 73: 52-57.

8. Sarela AI, Guthrie JA, Seymour MT, Ride E, Guillou PJ, O'Riordain DS. Non-operative management of primary tumour in patients with incurable stage IV colorectal cancer. Br J Surg 2001; 8: 1352-1356.

9. Greene FL, Page DL, Fleming ID. AJCC Cancer Staging Manual. $6^{\text {th }}$ Edition. New York, NY, Springer-Verlag, 2002.

10. Ruo L, Gougoutas C, Paty PB, Guillem JG, Cohen AM, Wong WD. Elective bowel resection for incurable stage IV colorectal cancer: prognostic variables for asymptomatic patients. J Am Coll Surg 2003; 196: 722-728.

11. Costi R, Mazzeo A, Di Mauro D, Veronesi L, Sansebastiano G, Violi V, et al. Palliative resection of colorrectal cancer: Does it prolong survival? Ann Surg Oncol 2007; 14: 2567-2576.

12. Yun HR, Lee WY, Lee OS, Cho YB, Yun SH, Chun $\mathrm{H}-\mathrm{K}$. The prognostic factors of stage IV colorrectal cancer and assessment of proper treatment according to the patient's status. Int J Colorectal Dis 2007; 22: 1301-1310.

13. Bannura G, Cumsille MA, Contreras J, Barrera A, Soto D, Melo C. CEA preoperatorio como factor pronóstico independiente en cáncer de colon y recto. Rev Méd Chile 2004; 132: 691-700.

14. Cook AD, Single R, McCahill LE. Surgical resection of primary tumors in patients who present with stage IV colorectal cancer: An analysis of surveillance, epidemiology, and end results data, 1988 to 2000 (SEER). Ann Surg Oncol 2005; 12: 637-645.

15. Konyalian VR, Rosing DK, Haukoos JS, Dixon MR, Sinow R, Bhaheetharan S, et al. The role of primary tumour resection in patients with stage IV colorectal 
cancer. Colorectal Dis 2006; 9: 430-437.

16. Liu SK, Church JM, Lavery IC, Fazio VW. Operation in patients with incurable colon cancer-is it worthwhile? Dis Colon Rectum 1997; 40: 11-14.

17. Scoggins CR, Meszoely IM, Blanke CD, Beauchamp $\mathrm{RD}$, Leach SD. Non-operative management of primary colorectal cancer in patients with stage IV disease. Ann Surg Oncol 1999; 6: 651-657.

18. Carne PW, Frye JN, Robertson GM, Frizelle FA. Stents or open operation for palliation of colorectal cancer: a retrospective, cohort study of perioperative outcome and long-term survival. Dis Colon Rectum 2004; 47: 1455-1461.

19. Beham A, Rentsch M, Pülmann K, Mantouvalou L, Spatz H, Schlitt HJ, et al. Survival benefit in patients after palliative resection versus non-resection colon cancer surgery. World J Gastroenterol 2006; 12: 66346638 .

20. Eisenberger A, Whelan RL, Neugut AI. Survival and symptomatic benefit from palliative primary tumor resection in patients with metastatic colorectal cancer: a review. Int J Colorectal Dis 2008; 23: 559-568. 